EU-Agrarpolitik

\section{Richtungsänderung verweigert}

Die Gemeinsame Agrarpolitik der EU (GAP) setzt mit rund $40 \%$ der EU-Haushaltsmittel entscheidende Anreize, wie die Agrarproduktion in der EU erfolgt. Sie ist schon seit langem in der Kritik, weil sie zum Klimawandel und zum Biodiversitätsverlust beiträgt und hinsichtlich der Einkommenssicherung der Landwirt*innen nicht die Bedürftigen erreicht. In Deutschland verursacht der Agrarsektor rund $12 \%$ der klimarelevanten Treibhausgasemissionen (inklusive der Emissionen aus Landnutzungswandel), ohne dass die Landwirtschaft bisher in nationale Minderungsstrategien einbezogen ist. Laut Naturzustandsbericht des Bundesamts für Naturschutz befinden sich $70 \%$ aller Flächen in Deutschland in einem schlechten ökologischen Zustand. Einen großen Anteil betreffen die Agrarökosysteme (Äcker und Grünland), die etwas mehr als die Hälfte der Fläche Deutschlands ausmachen. Der Verlust der biologischen Vielfalt schreitet voran, wenn nicht grundlegende Korrekturen in der Agrarpolitik vorgenommen werden. Wichtigster Faktor ist dabei die GAP.

Die Rufe nach einer Umkehr der GAP ertönen nicht nur von Umweltverbänden und Naturschutz. Auch von wissenschaftlicher Seite wurde belegt, dass sich die GAP höchst negativ auf Umwelt und Gesellschaft auswirkt. Zwei jüngst erschienene Studien der Leopoldina haben gezeigt, wie zerstörerisch die Effekte der heutigen Agrarproduktion und der Form unserer Ernährung (vor allem zu hoher Fleischkonsum) sind. Eine Auswertung von mehr als 600 wissenschaftlichen Studien hat zudem gezeigt, dass die GAP nicht nur umweltbezogen weitgehend versagt, sondern auch sozialen Ansprüchen nur sehr unvollkommen gerecht wird und im Gegensatz zu den Nachhaltigkeitszielen der Vereinten Nationen steht. Dies liegt vor allem daran, dass die Agrarsubventionen aus der ersten Säule der GAP mit mehr als $80 \%$ der Mittel nur mit schwachen Umweltzielen versehen und ohne Erfolgskontrolle pauschal an die Flächen gebunden sind. Ein großer Teil der Mittel kommt am Ende nur wenigen Betrieben zugute.

Der neue Europäische Green Deal sollte eine Wende bringen. Mit der „Farm to Fork"-Strategie als wichtigem Bestandteil sollte unter anderem der Weg hin zu mehr Klimaneutralität und einem höheren Schutzniveau im Biodiversitäts- und Naturschutz eingeleitet werden. Die ambitionierten Vorschläge setzen dabei auf die Mitwirkung aller

(C) Der/die Autor(en) 2020. Open Access: Dieser Artikel wird unter der Creative Commons Namensnennung 4.0 International Lizenz (https:// creativecommons.org/licenses/by/4.0/deed.de) veröffentlicht.

Open Access wird durch die ZBW - Leibniz-Informationszentrum Wirtschaft gefördert.
Akteur*innen nicht nur auf EU-Ebene, sondern auch auf der Ebene der Mitgliedstaaten. Doch die Richtungsänderung in der GAP ist nicht eingetreten. Im Gegenteil: Die Ende Oktober 2020 verabschiedeten Beschlüsse folgen eher einem „Ein Schritt vor, zwei Schritte zurück". Viele Einzelregelungen stehen einer notwendigen Umkehr entgegen. Schon der Vorschlag der Kommission war angesichts der großen Herausforderungen in der Landwirtschaft und der ärmlichen Performance der bisherigen GAP, die im Übrigen sogar vom Europäischen Rechnungshof kritisiert worden war, eine einzige Enttäuschung. Durch die Verhandlungen im Rat (unter Vorsitz der deutschen Landwirtschaftsministerin Julia Klöckner) und den Abstimmungen mit dem EU-Parlament wurde dann letztlich lediglich ein „Weiter so" beschlossen.

Die auf Flächen bezogene 1. Säule der GAP macht weiterhin den Löwenanteil der EU-Agrarfinanzierung aus, wie bisher. Eine Stärkung der Umwelt- und Klimaziele der 2. Säule der GAP bleibt aus. Dass die Mittel der 1. Säule nunmehr zu $30 \%$ an „Eco-Schemes“ gebunden sind, verschleiert, dass die Maßnahmen sehr schwach sind und auf Freiwilligkeit beruhen. Von Erfolgskontrolle keine Spur. Der Vorschlag, $10 \%$ der Flächen für Artenvielfalt aus der landwirtschaftlichen Nutzung herauszunehmen, wird nur als "Anregung“ formuliert. Es gibt weder Ziele zur weiteren Reduktion des Pestizid- und Düngemitteleintrags, noch gibt es Ziele zur Ausweitung des Ökolandbaus. Die Pariser Klimaziele werden zwar in die GAP aufgenommen, doch das Ziel einer konkreten Emissionsreduktion (30\% bis 2027) wurde abgelehnt. Es wird zudem hinsichtlich der Ausgestaltung der Programme viel Entscheidungsspielraum auf die Mitgliedstaaten verlagert. Hier steht zu befürchten, dass der Schutz der heimischen Landwirtschaft aus Wettbewerbsgründen eine weitere Abschwächung der Umweltstandards nach sich zieht.

Dass die deutsche Landwirtschaftsministerin in der jetzt beschlossenen GAP einen „Systemwechsel“ sieht, ist reine Augenwischerei und wieder einmal Ergebnis symbolischer Politik. Dass die Interessenvertretungen aus der Landwirtschaft die erreichten Kompromisse begrüßen, ist nicht überraschend und zeigt einmal mehr, dass es innen allein um die Interessen der großen Industrieproduzent*innen und reichen Landbesitzer*innen geht. Ob im Zuge der Umsetzung der GAP in Deutschland die nunmehr größeren nationalen Spielräume für eine Richtungsänderung genutzt werden, kann man angesichts der Dominanz der Partikularinteressen durchaus bezweifeln. Vielleicht gelingt es in einigen Bundesländern, wenigstens einige Schritte in diese Richtung zu tun.

Bernd Hansjürgens Helmholtz-Zentrum für Umweltforschung - UFZ bernd.hansjuergens@ufz.de 